\title{
CPAP and/or NIV in cardiogenic pulmonary oedema
}

\section{T. Köhnlein}

Hannover Medical School

Dept of Respiratory Medicine

Hannover

Germany

koehnlein.thomas@mh-hannover.de

Provenance

Adapted from an ERS School Course

Competing interests

None declared

\section{Summary}

Heart failure is a complex topic which poses difficult decisions for ventilator specialists. Currently available therapies are mechanical ventilation and standard therapy with oxygen or medical intervention. Ventilator specialists face a difficult decision when choosing which of these to use as the evidence for each is not conclusive. The primary aim of the chosen treatment is to improve oxygenation and respiratory acidosis and reduce the work of breathing and dyspnoea. In addition, another goal of treatment is an improvement of cardiac performance. To date, it is difficult to recommend which available treatment is the best at achieving these goals and it is recommended that individual treatment for each patient should be based on their specific needs and circumstances.

\section{Theory of mechanical ventilation}

To use continuous positive airway pressure (CPAP) or noninvasive ventilation (NIV) effectively it is important to understand the interplay between breathing and cardiac function.

\section{Effect of normal breathing on cardiac function}

In healthy individuals, inspiration causes transpleural pressure to become negative, which in turn causes intrathoracic pressure to become negative, leading to an increase in venous return to the heart. An increase in venous return causes a larger preload, increased pressure in the right atrium and increased end diastolic filling pressure in the right ventricle. On the left side of the heart, the negative intrathoracic pressure causes an increase in left ventricular afterload leading to a higher cardiac workload.

\section{Effect of positive pressure in healthy individuals}

The application of positive pressure has an impact on the complex pressure and circulation interplay in the chest, although the exact mechanism has not been determined. In healthy individuals, the use of positive pressure causes
HERMES syllabus link: modules B.14.2, E.1.6 
Figure 1

The effects of mechanical ventilation on the central haemodynamics in a healthy individual.

\section{Figure 2}

Models of pressure gradients during normal spontaneous breathing and with the use of CPAP in a healthy individual.

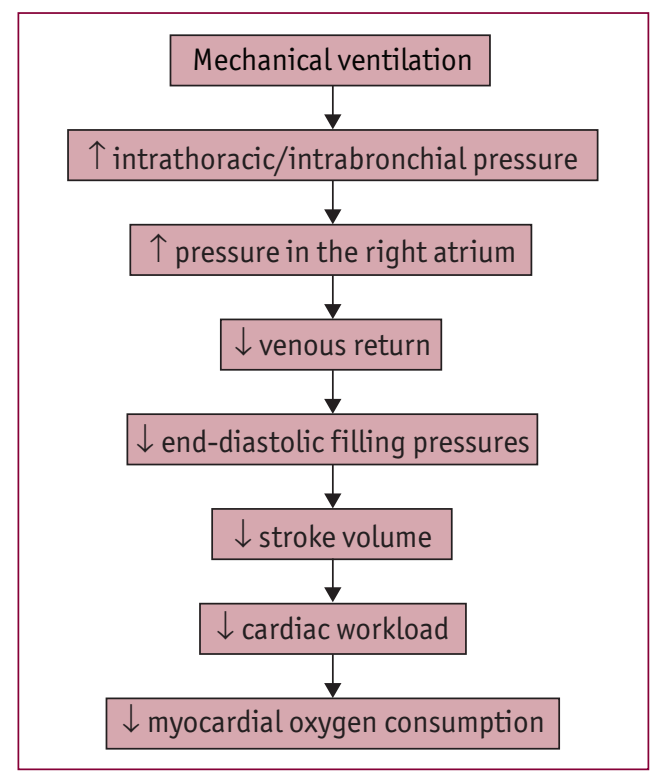

an increase in intrathoracic pressure, which leads to a decrease in venous return, a decrease in cardiac filling pressures, causing a reduction in myocardial work load (figure 1).

It is thought that the effect of NIV and CPAP therapy in patients with cardiogenic pulmonary oedema is due to a combined reduction of cardiac pre- and afterload. This is a similar effect to that caused by angiotensin-converting enzyme inhibitors or nitrates, but much faster [1]. Elevation of the intrathoracic pressure diminishes the cardiac minute volume in healthy individuals, but in patients with left ventricular insufficiency, the stroke volume and the cardiac index can also be improved.

\section{Mechanical ventilation in real life}

The treatment for a patient presenting with cardiac failure with pulmonary oedema is currently recommended to be either CPAP between 8 and 12 mbar or NIV in assisted mode with a short rise time and high flow to achieve a high inspiratory pressure (20 mbar) and a high positive end-expiratory pressure (6-10 mbar). Facial masks or the helmet are the interfaces of choice. Although these recommendations are common practice there is not real evidence to suggest that this is the correct procedure in patients with heart failure.

Several physiological studies have examined the benefit of using positive pressure for heart failure patients. A small study by KotANIDOU et al. [1] included 12 healthy volunteers and 12 patients with severe heart failure and studied the effect of applying 5, 15 and 20 mbar of CPAP on cardiac output. In the healthy volunteers, cardiac output decreased due to an increase in intrathoracic pressure leading to a decrease in venous return.

However, the same result was not found in heart failure patients, in fact, cardiac output remained steady on all levels of CPAP, with a slight increase when intrathoracic pressure was increased up to 20 mbar of CPAP.

The haemodynamic effects of CPAP and NIV were evaluated in 10 patients with acute deterioration of cardiac function in a study published in a)

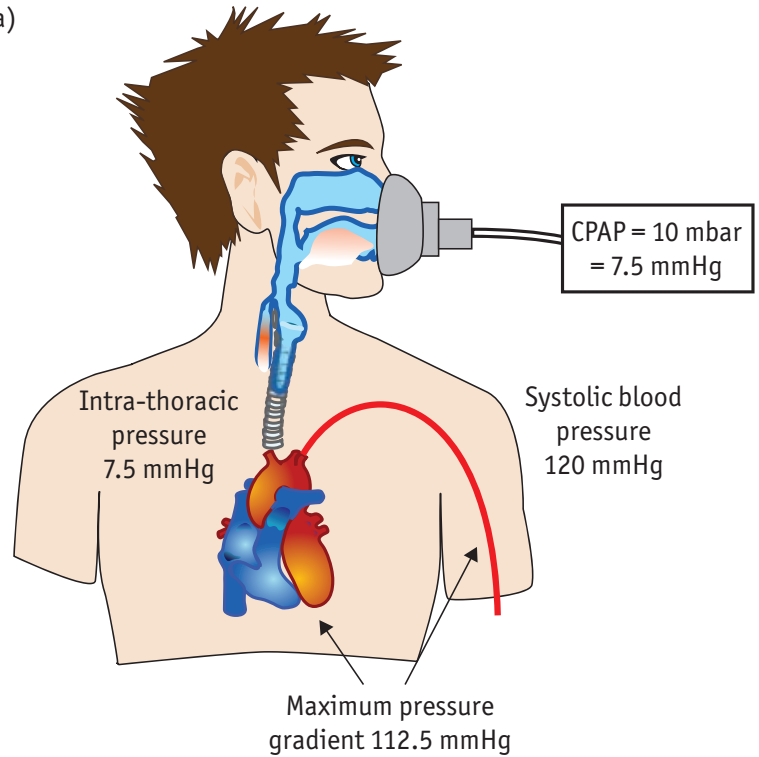

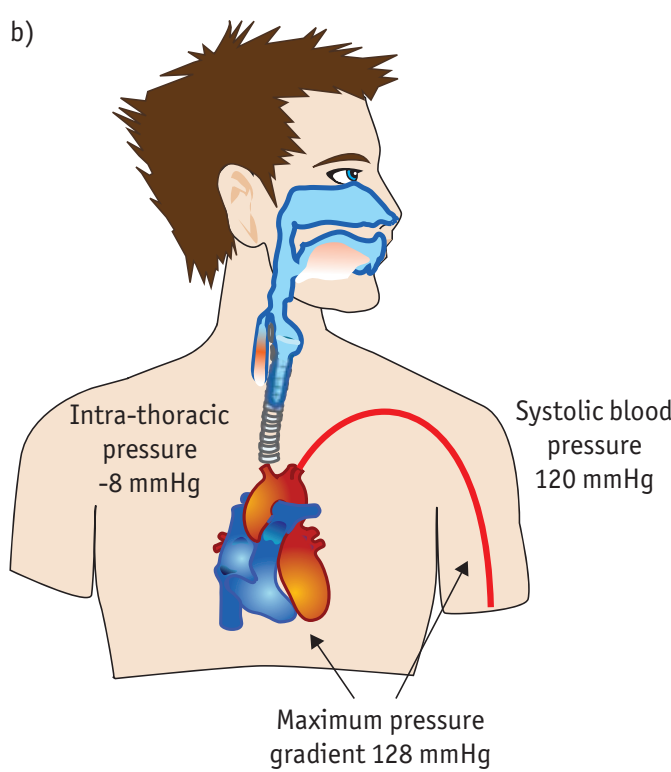


2002 [4]. CPAP of 10 mbar or bi-level positive airway pressure (BiPAP) of 15/5 mbar was applied over $30 \mathrm{~min}$ in the crossover study. The primary end-point was changes functional mitral regurgitation (MR). After 30 min of CPAP and BiPAP, MR decreased significantly in all 10 patients demonstrating that both methods were effective at improving ejection fraction and reducing MR in patients with exacerbations of severe chronic heart failure.

\section{NIV versus standard therapy}

In 1998, a meta-analysis compared studies examining the addition of CPAP or NIV to standard therapy on mortality rates and the need for intubation [5]. The application of CPAP versus standard treatment in acute pulmonary oedema was shown to reduce mortality on average by $6 \%$ and intubation rates by $26 \%$ (table 1 ). The study concluded that evidence was in favour of using CPAP in patients with cardiogenic pulmonary oedema, although potential side effects and long-term problems with the technique were not ruled out.

In 2003, a large multicentre study performed in the emergency room included 130 patients with acute respiratory failure who were randomised to receive either medical therapy including oxygen or medical therapy, oxygen and NIV [6]. The primary end-point of the study was the need for intubation with a secondary end-point of physiological changes. In total, 65 patients were allocated NIV, 16 of these 65 patients had to be intubated and 49 patients were treated successfully without the need for intubation. In comparison, of the 65 patients included in the standard therapy arm 13 patients were intubated and 52 patients were treated successfully without intubation. The NIV group was found to have a significantly better improvement of the oxygenation in the first $3 \mathrm{~h}$ after start of treatment. In addition, respiratory rate, dyspnoea score, blood pressure and heart rate showed a significant improvement in the first hours after the initiation of NIV treatment. Therefore, NIV was superior at providing a more rapid improvement in oxygenation and dyspnoea compared with standard medical therapy alone. However, NIV did not decrease the overall use of intubation, mortality or adverse events which were equally distributed between the two groups.

Until recently, multiple clinical trials revealed that physiological parameters improve with the use of CPAP or NIV, in addition endotracheal intubation rates and shortterm mortality may be reduced. However, a recent large multicentre clinical effectiveness trial [7] failed to show any clear overall benefit of CPAP or NIV for patients with severe cardiopulmonary oedema, despite some improvement in physiological parameters and patient symptoms. The study included 1,069 patients assigned to CPAP, NIV or standard therapy with a primary end-point of death within 7 days of treatment [7]. It was found that there was no significant difference in 7-day mortality between patients receiving standard oxygen therapy and those receiving CPAP or NIV. Moreover, there was no significant difference between intubation rates. However, similar to the NAVA et al. [6] study, NIV was associated with greater improvements in dyspnoea, heart rate, acidosis and hypercapnia $1 \mathrm{~h}$ after treatment.

\section{Recommendations for clinical treatment}

NIV has been seen to have beneficial effects within the first few hours of treatment; however, the outcome after days or longer seems to be more or less comparable with that of standard therapy with oxygen. Therefore, CPAP and NIV

Table 1 The effect of CPAP on hospital mortality in cardiogenic pulmonary oedema patients

\begin{tabular}{|c|c|c|c|c|c|c|c|c|}
\hline & \multicolumn{3}{|c|}{ CPAP } & \multicolumn{3}{|c|}{ Standard treatment } & \multirow{2}{*}{$\begin{array}{l}\text { Risk reduction in } \\
\text { mortality } \%\end{array}$} & \multirow{2}{*}{$\begin{array}{l}\text { Risk reduction for } \\
\text { intubation rate } \%\end{array}$} \\
\hline & Total & Intubated & Mortality & Total & Intubated & Mortality & & \\
\hline BERSTEN et al. 1991 & 19 & 0 & 2 & 20 & 7 & 4 & -9.5 & -35 \\
\hline Lin et al. & 50 & 8 & 4 & 50 & 18 & 6 & -4 & -20 \\
\hline
\end{tabular}


in combination with standard medical treatment are good options in the acute setting for cardiogenic oedema patients as they will provide more immediate relief compared to the patients with standard therapy. What to do in the clinical setting is still a difficult decision, as patients with heart failure generally have different associated diseases, for example pulmonary disease, chronic obstructive pulmonary disease and effects from smoking. Therefore, it is important to make treatment decisions based on the individual and what the risks and benefits are in that particular case.

CPAP can be considered as first line treatment in patients with acute cardiogenic pulmonary oedema. CPAP equipment is, in general, not very complex and cheap. In addition, a number of simple systems allow the delivery of 100\% oxygen. In the majority of other patients, medical therapy should be instigated as the primary treatment of acute cardiogenic pulmonary oedema, and CPAP or NIV should be applied in patients who have significant respiratory distress and failure, or those not improving with standard medical treatment.

Until today, there is still some concern about myocardial infarctions during NIV in patients with advanced cardiac insufficiency. This concern was prompted by a paper by MeHTA et al. [8], published in 1997 that compared CPAP and NIV in patients with acute cardiogenic pulmonary oedema. The study found that the rate of myocardial infarction was higher in the NIV group compared with the CPAP group. All later performed studies with much larger cohorts could not confirm these findings. Myocardial infarction rates are low, independent of CPAP or NIV application and both modes of ventilation are not contraindicated in patients with angina. However, more caution should be taken in patients with severe cardiac arrhythmia who might need defibrillation.

\section{Pre-clinical NIV}

It has recently been thought that NIV could be applied in the preclinical setting in ambulances or in patients' homes to relieve symptoms of dyspnoea immediately. Several devices with NIV modes are becoming available that could be used by paramedics in the preclinical setting. However, these devices are currently very expensive and are not widespread.

There are several systems on the market, for example the Boussignac CPAP system (Vitaid Ltd, Toronto, Canada), which uses microchannels to force oxygen through the system to accelerate the molecules which then get deflected into a central area where they collide creating positive pressure. The system is powerful enough to produce a CPAP of $\sim 8 \mathrm{~mL}$ of water and there has been firm data to suggest that the device does work [9].

Currently there are studies going on in Germany to examine the benefits of using portable CPAP devices that can be used before the patient reaches the hospital. Preliminary results from one of these studies have been reported, which included 100 patients treated with the device who were later confirmed to have pulmonary oedema or chronic obstructive pulmonary disease [10]. The report has documented the opinion of the paramedic or doctor at the scene of the emergency using the ventilation systems. In general, a reduction in respiratory rate, blood pressure and heart rate was observed after treatment with the CPAP device. The opinion of the paramedic or doctor was taken into account and in total $52 \%$ rated the clinical situation after using the CPAP device much improved and 40\% rated it improved. In addition, $59 \%$ of medical professionals avoided intubation after using the device and 32\% thought intubation was not necessary after CPAP treatment. Therefore, this is an interesting topic which should be considered an option for the development of NIV treatment, especially in heart failure patients. 


\section{References}

1. Kotanidou A, Armaganidis A, Zakynthinos S, Mavrommatis A, Roussos C. Changes in thoracopulmonary compliance and hemodynamic effects of positive end-expiratory pressure in patients with or without heart failure. $J$ Crit Care 1997; 12: 101-110.

2. Leithner C, Podolsky A, Globits S, et al. Magnetic resonance imaging of the heart during positive end-expiratory pressure ventilation in normal subjects. Crit Care Med 1994; 22: 426-432.

3. Mehta S, Liu PP, Fitzgerald FS, Allidina YK, Bradley D. Effects of continuous positive airway pressure on cardiac volumes in patients with ischemic ad dilated cardiomyopathy. Am J Respir Crit Care Med 2000; 161: 128-134.

4. Bellone A, Barbieri A, Ricci C, et al. Acute effects of non-invasive ventilatory support on functional mitral regurgitation in patients with exacerbation of congestive heart failure. Intensive Care Med 2002; 28: 1348-1350.

5. Pang D, Keenan SP, Cook DJ, Sibbald WJ. The effect of positive pressure airway support on mortality and the need for intubation in cardiogenic pulmonary edmea. Chest 1998; 114: 1185-1192.

6. Nava S, Carbone G, DiBattista N, et al. Noninvasive ventilation in cardiogenic pulmonary edema. Am J Respir Crit Care Med 2003; 168: 1432-1437.

7. Gray A, Goodacre S, Newby DE, et al. Noninvasive ventilation in acute cardiogenic pulmonary edema. $N$ Engl J Med 2008; 359: 142-151.

8. Mehta S, Jay GD, Woolard RH, et al. Randomized, prospective trial of bilevel versus continuous positive airway pressure in acute pulmonary edema. Crit Care Med 1997; 25: 620-628.

9. Cigada M, Elia G, Giacomini M, et al. Novel indications for the Boussignac CPAP valve. Intensive Care Med 2007; 33: 374-375.

10. Jerrentrup A, Sattler A, Canisius C, Kill C, Vogelmeier C. Präklinische CPAP-Beatmung bei akuter respiratorischer Insuffizienz [Preclinical CPAP ventialtion for acute respiratory insufficiency]. Oral presentation at the annual meeting of Deutsche Gesellschaft für Pneumologie und Beatmungsmedizin e. V., Mannheim, 2007. 